\title{
SUPERIOR
}

\section{EVOLUTION OF CONSCIOUSNESS II}

\author{
Richard A. Mould* \\ Department of Physics and Astronomy, State University of New York, Stony Brook, USA \\ Received April 14, 2009; accepted April 29, 2009
}

\begin{abstract}
A mechanism that allows the conscious experience of 'pain' to be a modifier of behavior and hence a factor in evolutionary development, is proposed in a previous paper. There are a number of other experiences called 'anchor' experiences that perform the same function. Several of these are identified and distinguished from non-anchor experiences.
\end{abstract}

Key words: Anchor Experience; Consciousness; Parallel Principle; Psychophysical Parallelism

\section{INTRODUCTION}

In a previous paper the author proposes a way of shaping the conscious states of an evolving species through an interaction between conscious states and physical states (Mould, 2009). Interaction is necessary to insure that both kinds of states mirror one another throughout their evolution. They follow a 'parallel principle' that is implicit in von Neumann's psycho-physical parallelism (Mould, 2001; von Neumann, 1955).

The example is that of a fish that is a successful automaton throughout most of its evolution. At some point pain consciousness is introduced through a genetic mutation, where this supposedly happens when the fish makes contact with the toxic tentacles of a jellyfish. Pain consciousness is always assumed to have a represive effect on the attending behavior; and as a result, escape is preferred over tentacle contact. An incorrect behavioral response (in which pain represses an escape from the jellyfish tentacles) is allowed by this mechanism, but that would threaten the survival of the species. Natural selection therefore supports escape. Although an automation might evolve in a satisfactory way all by itself (i.e., without the aid of consciousness), it is assumed that the purpose of consciousness operating in tandem with biological automation is to benefit the species by furthering its evolution.

In this paper the above development is initially extended by imagining that the fish not only experiences pain when it makes contact with the tentacles, but it also becomes consciously aware of the approaching jellyfish through a new experience - visual consciousness. The fish associates this new experience with the pain of tentacle contact, and consequently it learns another new

*E-mail:rmould@notes.cc.sunysb.edu experience - fear consciousness. As in the case of pain, natural selection couples fearful experience with an appropriate behavioral response. That is, fear is assumed to have a repressive effect on the attending behavior as does pain. Both are conscious experiences that have a direct causal influence of a specific kind on behavioral and evolutionary development.

Introducing pain consciousness is one evolutionary step using the proposed mechanism. Introducing fear is another evolutionary step that can use the same mechanism. However, sequencing these two experiences and filling in the gaps between them requires steps that are not obviously accommodated by the proposed mechanism. There are five separate steps from visual contact to avoidance.

The fish

(1) evolves a visual image of the jellyfishes tentacles.

(2) learns to associate this image with contact.

(3) learns to associate (1) and (2) with 'pain'.

(4) associates steps (1-3) with a new experience - 'fear'

(5) learns to avoid the jellyfish through fear, inasmuch as fear can also drives evolution by supporting avoidance behavior.

Step (1) presumes a prior (pre-conscious) evolution of photon receptors, such as eyes, optical nerves, etc. The visual awareness in step (1) is a separate 'additional' development that serves no evolutionary purpose if it does not include step (5). That's because visual experience cannot directly affect behavior and evolution in the same way as pain and fear. Although the five steps taken together may provide an additional evolutionary 
advantage to the creature, their occurring simultaneously as a single step seems unlikely. The five-step scenario is therefore improbable.

\section{ANCHORS}

I call conscious experiences anchors if they have a direct influence on behavior. Pain is an anchor. Fear is another anchor. An anchor is an experience that has an intrinsic motivational direction, thereby having a definite positive or negative effect on the behavior and hence evolution. We recognize them as emotions that drive us one way or another. Without anchors that provide the causal feedback from consciousness to physiology, evolutionary parallelism would be impossible. In the above five-step process 'vision' is not an anchor. It has no intrinsic motivational power that can affect behavior. It acquires importance only as a connector between the anchors (or emotions) that drive evolution. In the above case, it connects the anchor 'pain' with the anchor 'fear', where the latter is a direct modifier of behavior that stands in for pain in the context provided by visual experience.

The improbability of steps (1-5) can possibly be overcome by increasing the number of situations in which vision can play a connecting role, and by increasing the number of anchors that are involved. In order to justify the introduction of an intrinsi-cally unmotivated experience like vision, a wide variety of anchor experiences must be connected by it in a wide variety of life situations.

\section{OTHER ANCHORS}

Hunger is a believable anchor inasmuch as it has an intrinsic motivational authority. It is a conscious experience that we imagine has a positive effect on the hunting behavior at a time when the creature needs to be actively looking for food. Taste is another possible anchor that provides intrinsic motivation. It too can have a positive or negative polarity. It has a positive influence on eating behavior when the organism benefits from the food in question and a negative influence when the food is harmful. As with all of the polarities established by anchors, a wrong association (i.e., coupling a positive influence with harmful food) will threaten the survival of the species; so again, natural selection favors an appropriate association of subjective states with physiological states. Smell is still another experience that qualifies as an anchor, for it also has definite positive and negative motivating properties.

Perceptions such as sight, sound, and non-noceceptive touch are not generally motivating in a positive or negative direction. A possible exception for touch is noted in the last paragraph of the next section. Unlike taste or smell, there is nothing generally provocative about sight, sound, and ordinary touch that would prompt an instinctive yes or no reaction. This means that a con- scious creature can only respond to things seen, heard, or touched when an experiential context is provided by the anchors. The anchors then become embedded in perceptual interpretations that bring a unity to the entire psychological experience.

\section{ANCHOR CLUSTERS}

There are now six prospective anchor experiences: pleasure, pain, fear, hunger, taste, and smell, where each is capable of affecting the direction of the evolution of a species. There is quite a large gap between pain and fear as we have seen, but there is not a large gap between clusters that includes hunger, taste, and smell. So we can imagine an evolutionary scenario that begins with just these three in some combination.

Since the hunger for food and the smell of food very often occur simultaneously, it is likely that they will reinforce each other. If hunger represses inactive behavior, and if the right smell enhances active hunting behavior, then the two together should provide a powerful incentive to hunt. They can occur separately, and they certainly must have been introduced separately, but the pair function as a cluster in many situations.

Good smell and good taste might also become associated with each other at the time of a kill. Couple this with a fading of aversive hunger, and the combination is a compel-ling conscious experience. The introduction of taste as another evolutionary step will therefore cement this cluster of three.

Fear and smell consciousness might be aroused simultaneously in the presence of a predator, thereby bringing fear into the established cluster; and pleasure might make a connection to the cluster through taste, and possibly through smell.

The anchors hunger, taste, smell, fear, and pleasure therefore provide a cluster around which other anchors and other elements of consciousness can become attached. It is entirely possible that the construction of visual, auditory, and tactual images will first emerge around this cluster of anchor experiences rather than with pain alone as in steps (1-5). If while hunting and feeding the creature begins to have new experiences identifiable as sights, sounds, or touches, and if these are associated with the predators, prey, and other objects in the creature's environment, and if this association reinforces the anchor experiences within the cluster that directly influence behavior, then these newly introduced non-anchor experiences should be supportive of the species. As visual, acoustic, and tactual experiences become more and more sophisticated, they will allow the construction of images of the external world that are in accord with the psycho-physical parallelism. This construction does not have to be a single step in evolution. It could very well be a gradual process that is brought on at every point by established conscious clusters. The above five-step scenario would then be re- 
placed by a process that spreads step (1) out over the remaining four steps.

Other scenarios are possible that are centered on sex and/or mothering, both of which provide strongly positive motivations that have their roots in pre-conscious evolution. Indeed, any clearly directional instinct or emotion has the potential to become an experiential anchor; and any anchor or group of anchors has the potential to become a cluster of consciousness that can support other (non-anchor) subjective elements, images, and ultimately ideas about the physical world. For primitive conscious creatures, anchors and non-anchors alike support a behavior that has already been established by the pre-conscious automaton.

Sex and mothering especially provide a context that allows the otherwise neutral experience of 'touch' to play a more motivational (anchor-like) role. Touch may then help motivate a world picture that goes beyond a narrow sexual or mothering context. While touch is primarily perceptual, it is also much related to both pleasure and pain. So while it is primarily non-anchor, it often functions in an anchor capacity in a sensual situation. No experience is absolutely an anchor or nonanchor experience, and touch may best illustrate the ambiguity of that separation. Generally speaking however, the emerging pattern suggests that instincts or emotions are anchors, and that conscious perceptions (images and ideas) are not anchors.

\section{ANCHOR/IMAGE INTERDEPENDENCE}

Anger is another anchor experience. It plays a role in aggressive behavior having to do with defending turf or fighting for dominance within a herd. It is an emotion that emerges as a separate evolutionary step, giving us a total of seven independent anchors.

However, there is a curious interdependence between anger and fear that is reflected in the body's fight or flight mechanism. The creature must evaluate a threatening situa-tion and decide whether it is best to make a stand, or quit the field. It must decide if it should give in to its fear, or get angry. This is a matter of deciding which anchor to engage, and hence, which behavior to enhance. The implication is that the images that are created in conjunction with established anchors can subsequently be used to evaluate an objective situation, and then decide in favor of one anchor or another. Most species display an anchor/image interdependence of some kind, and when it reaches the conscious level the fight or flight mechanism - becoming the anger or fear experience - is probably the most primitive of this genre.

\section{REFERENCES}

Mould, R. A. (2009). Evolution of Consciousness I. Activitas Nervosa Superior, 51, 83-86 (present issue).

Mould, R.A. (2001). The Parallel Principle, arXiv:quant$\mathrm{ph} / 011109$

von Neumann, J. (1955). Mathematical Foundations of Quantum Mechanics. Princeton: Princeton University Press. 